\title{
LOW-COST THERMAL MEMS GYROSCOPE
}

\author{
A.M. Leung \\ School of Engineering Science, Simon Fraser University, Burnaby, BC, Canada
}

\begin{abstract}
This paper describes a new MEMS gyroscope (gyro) with a simple device structure. Its operation is based on the Coriolis effect on an oscillatory gas stream. The oscillatory motion is created by two heaters which are activated alternately. The fabrication of the thermal MEMS gyro is not equipment intensive or process critical, making this device a potential contender for the low-cost MEMS gyro market. A rudimentary prototype running at $4 \mathrm{~mW}$ heater power has shown a linearity of better than $1 \%$ with a $360 \%$ sull scale, and a noise density of $1.5 \% / \mathrm{s} / \mathrm{Hz}^{0.5}$.
\end{abstract}

\section{INTRODUCTION}

With very few exceptions, all commercial MEMS gyros measure Coriolis effects on a moving solid proof mass at resonance to determine angular rate $[1,2]$. Tight control of proof mass dimensions is required to achieve good performance, particularly in the capacitance sensing type. The extremely low cost requirement of the large consumer market is a tremendous challenge for manufacturers of this type of MEMS gyroscopes, considering the fact that the design of resonator-based gyroscopes is a mature field. The work reported here explores the possibility of achieving lower cost using a fluidic angular sensing mechanism based on thermal principles.

Angular rate sensors with no vibrating solid proof mass are not new. An apparatus for measuring angular rate was disclosed in 1932 as a "turn indicator" [3]. It was built using traditional machined parts to detect moving fluid path deviation resulted from Coriolis acceleration. Improvements to this type of angular rate sensor include a miniature electric pump for moving fluid and temperature sensors for detecting fluid stream position [4]. While not as active as the vibrating mass MEMS gyro development, research efforts continue in fluidic angular rate sensor based on forced convection produced by a piezoelectric pump [5]. In spite of these efforts, the size, complexity and cost of these research devices have prevented them from becoming mainstream MEMS gyro contenders. Furthermore, these reported fluidic gyros use unidirectional flow which makes it impossible to cancel the linear acceleration effect [2]. The device described in this paper can achieve very low manufacture cost due to its simple device structure, and has a built-in linear acceleration effect cancellation capability to improve device performance.

\section{PRINCIPLE OF OPERATION}

The operating principle of the new thermal MEMS gyroscope is illustrated in Figure 1. In an enclosed space, two miniature filaments $\mathrm{H}_{\mathrm{T}}$ and $\mathrm{H}_{\mathrm{B}}$, or Top Heater and Bottom Heater, are placed on the line of symmetry between two temperature sensors, $\mathrm{T}_{\mathrm{L}}$ and $T_{R}$, or Left Temperature Sensor and Right Temperature Sensor. The Top Heater and Bottom Heater are activated alternately to move the gas between them. In Phase I of the device operation, shown in Figure 1(a), a current through $\mathrm{H}_{\mathrm{B}}$ 's filament heats it up while $\mathrm{H}_{\mathrm{T}}$ stays at the ambient temperature. As illustrated by the shaded circle, the gas in the vicinity of $\mathrm{H}_{B}$ heats up and expands. Before thermal equilibrium is reached, this gas expansion creates a moving gas stream, illustrated by the dotted arcs, towards $\mathrm{H}_{\mathrm{T}}$. With no rotation, i.e. angular rate $\Omega=0$, this gas stream moves along a symmetrical path between the two temperature sensors $\mathrm{T}_{\mathrm{L}}$ and $T_{R}$. This is indicated by the thick arrow in Figure 1(a). Due to symmetry, this gas movement produces no differential temperature
$\Delta T$. In Phase II when $\mathrm{H}_{\mathrm{T}}$ heats up, as shown in Figure 1(b), the direction of gas flow reverses but differential temperature output $\Delta T$ remains at zero because of symmetry and no rotation.

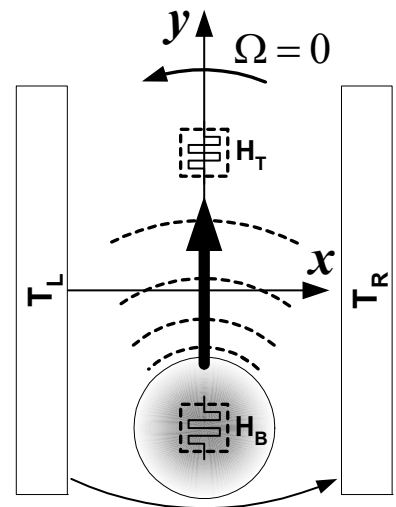

Phase I $\Delta T=0$

(a)

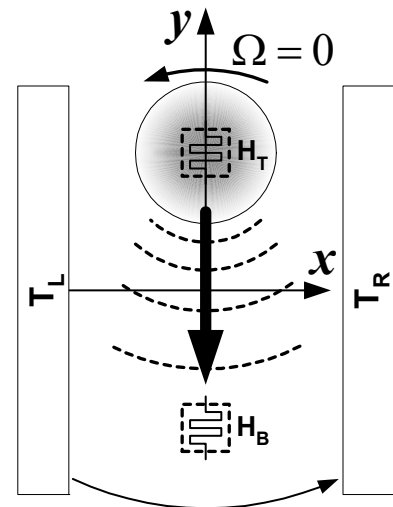

Phase II $\Delta T=0$

(b)
Figure 1: Thermal gyroscope operation with no rotation. Differential temperate output $\Delta T$ is zero in both Phase $I$ (a) and Phase II (b).

When the device is rotated, Coriolis acceleration acts on the moving gas stream and disturbs its symmetrical path. In Phase I of the device operation, this is illustrated by the thick arrow in Figure 2(a). The higher temperature gas stream moves towards $T_{R}$ and generates a time dependent positive $\Delta T(t)$. Phase II of the device operation is shown in Figure 2(b). When $\mathrm{H}_{\mathrm{T}}$ is activated instead of $\mathrm{H}_{\mathrm{B}}$, the direction of the gas movement reverses. This creates a negative $\Delta T(t)$. By reversing the polarity of $\Delta T(t)$ synchronous to the activation of $\mathrm{H}_{\mathrm{B}}$ and $\mathrm{H}_{\mathrm{T}}$, an output signal proportional to the angular rate and direction can be obtained. This synchronous detection method also suppresses the linear acceleration signal, provided the device is symmetrical.

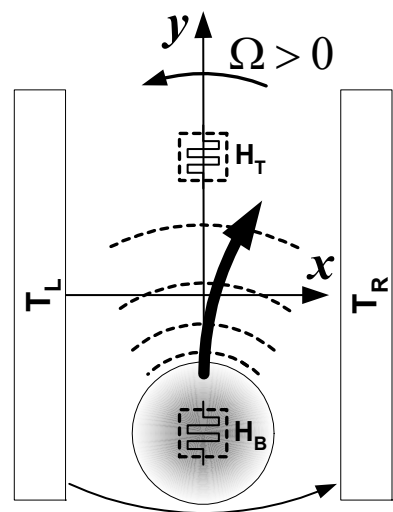

Phase I $\Delta T>0$

(a)

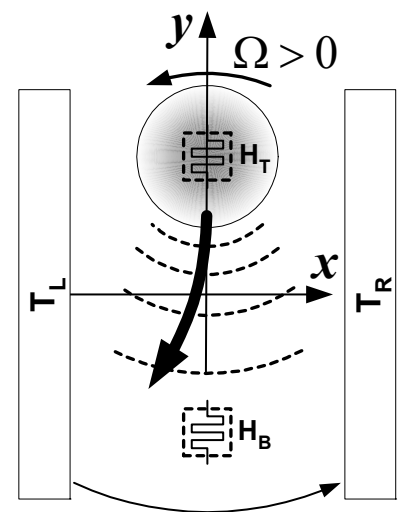

Phase II $\Delta T<0$ (b)

Figure 2: Thermal gyroscope operation with rotation. (a) Differential temperate output $\Delta T$ is positive in Phase I when $H_{B}$ is activated. (b) In Phase II when $H_{T}$ is activated, the gas stream reverses direction and produces a negative $\Delta T$. 


\section{RESULTS}

\section{Device structure}

The construction of the MEMS thermal gyro is illustrated in Figure 3. It is fabricated using a 2 -mask process. A $0.4 \mu \mathrm{m}$ thick CVD oxide and a $0.5 \mu \mathrm{m}$ thick aluminum are deposited on the silicon substrate. The aluminum thin film is first patterned to form the bond pads, and the resistors for the heaters and the temperature sensors corresponding to $\mathrm{H}_{T}, \mathrm{H}_{\mathrm{B}}, \mathrm{T}_{\mathrm{L}}$ and $\mathrm{T}_{\mathrm{R}}$ shown in Figure 2 . The oxide layer is then patterned to define the widows for silicon etching. Isotropic dry etching using Xenon Difluoride [6] results in a $300 \mu \mathrm{m}$-deep cavity on the silicon substrate, and creates three suspended oxide bridges for the heaters and temperature sensors shown in Figure 3. The top view of the prototype thermal gyro is shown in Figure 4.

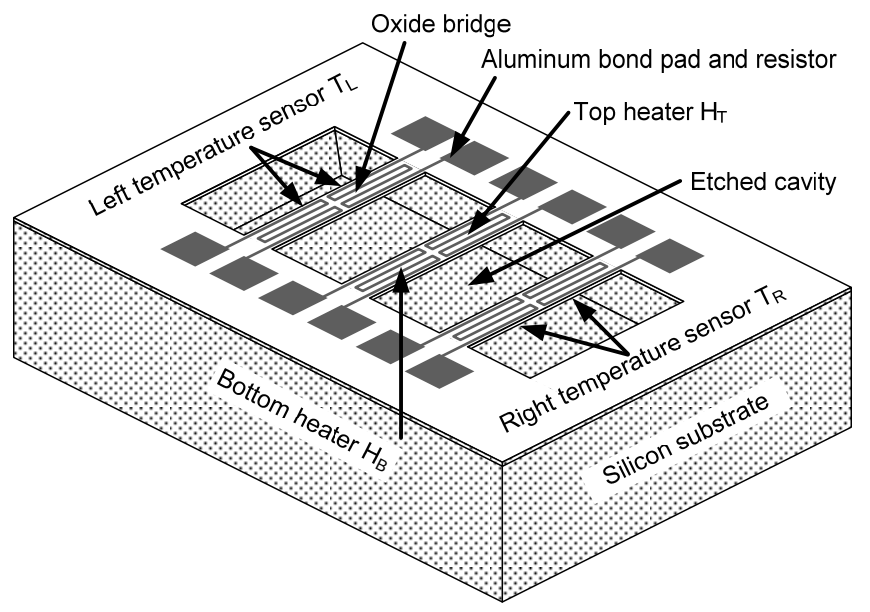

Figure 3: Thermal gyro device structure.

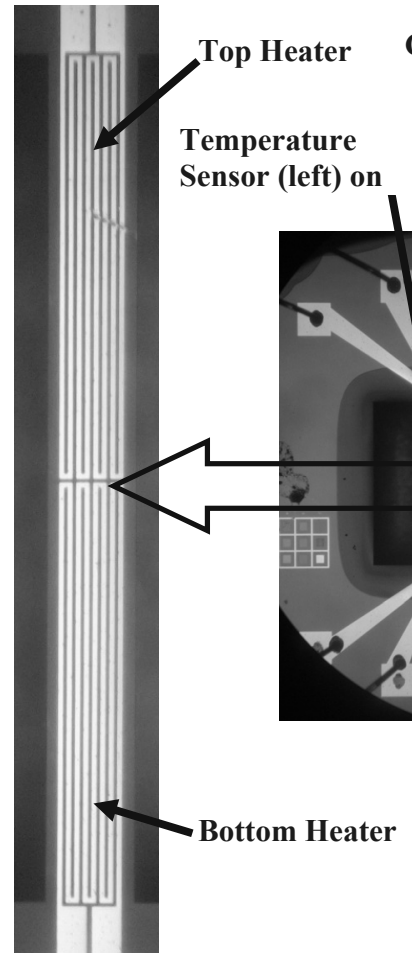

Figure 4: Top view of Thermal Gyro prototype.
To increase sensitivity, the thermal gyro is sealed in a 16-pin dual-in-line package, shown in Figure 5, which is filled with a high density sulfur hexafluoride gas. It is worthwhile to note that this thermal gyro device structure is practically identical to that of the thermal accelerometer reported more than a decade ago [7], except that the heater bridge is electrically divided to form two heaters.

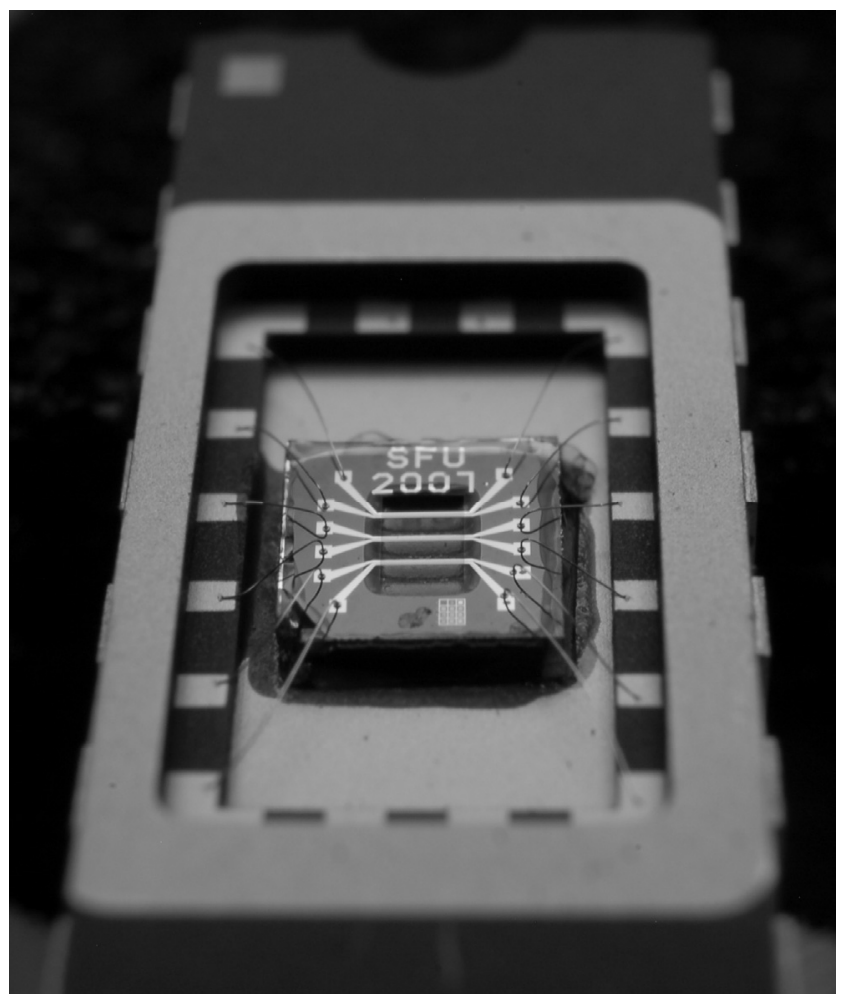

Figure 5: Thermal Gyro in 16-pin ceramic package. Wire bonding and cavity etching completed. It is ready to be sealed in $S F_{6}$.

\section{Prototype testing}

A simple test setup shown in Figure 6 is used to verify the functionality of the thermal gyro. A rotational platform for testing the thermal gyro is attached directly to the shaft of a servo motor. The thermal MEMS gyro, amplifiers, and signal processing circuits are assembled on a prototype board mounted on the rotational platform. Wire connections to stationary data recording equipment interfere with the platform's rotation. They are replaced by a battery power source and a short-range infrared (IR) data link consists of three IR light emitting diodes. The amplified thermal gyro signal is used to frequency modulate the IR light emitting diode current. A phase lock loop is used in the IR receiver to recover the analog thermal gyro signal.

In characterizing the thermal gyro prototype, the servo motor is programmed to rotate at rates from $-360^{\circ} / \mathrm{s}$ to $+360^{\circ} / \mathrm{s}$ with $90^{\circ} / \mathrm{s}$ step increments. At each rotational rate the thermal gyro output is recorded. The data of the thermal gyro output as a function of angular rate are summarized in Figure 7 . The testing setup specifics and measured results are summarized below:

$\begin{array}{ll}\text { Heater power: } & 4 \mathrm{~mW} \\ \text { Heater switching frequency: } & 33 \mathrm{~Hz} \\ \text { Signal bandwidth: } & 3 \mathrm{~Hz} \\ \text { Device sensitivity: } & 24 \mu \mathrm{V} / 360 / \mathrm{s} \\ \text { Noise: } & 1.5^{\circ} / \mathrm{s} / \mathrm{Hz}^{0.5}\end{array}$




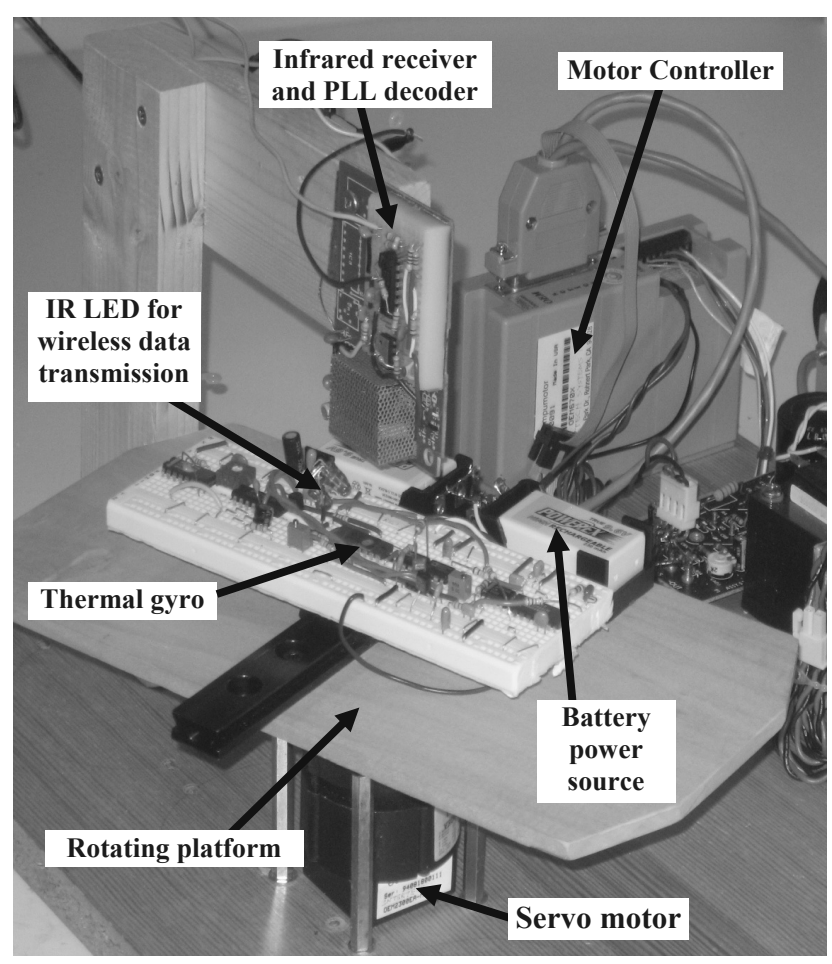

Figure 6: Experimental setup for Thermal Gyro prototype characterization.

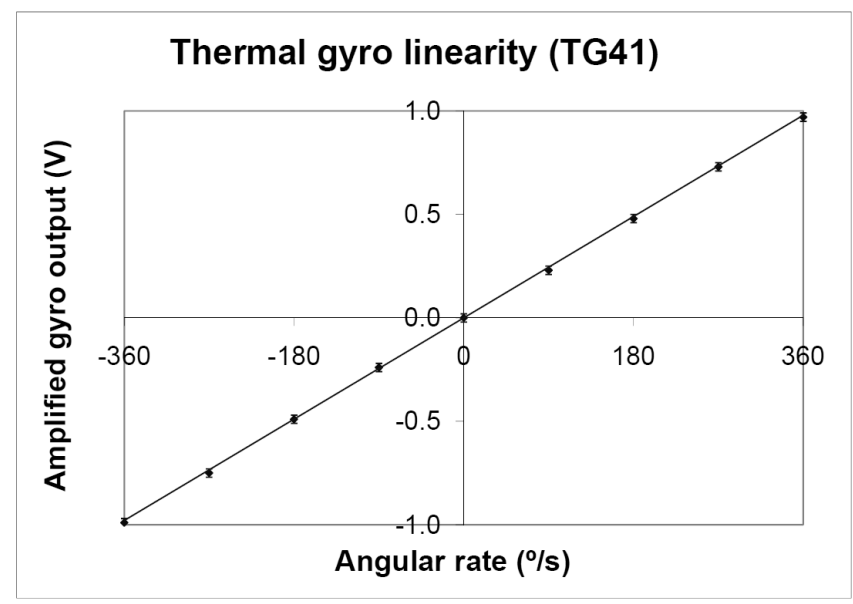

Figure 7: Thermal gyro output as a function of angular rate.

\section{Discussions}

The preliminary data collected from the thermal gyro prototype are surprisingly encouraging. Not only has the operational principal of this new thermal gyro validated by the prototype's functionality, a linearity of better than $1 \%$ for a $360 \% / \mathrm{S}$ full scale input is observed.

It is anticipated that the sensitivity of the thermal gyro can increase as the square of heater power. This can provide significant improvement of device performance. In our prototype, however, the heater power is limited to about $4 \mathrm{~mW}$ because of low electrical resistance of the aluminum heater resistor, and the limited current carrying capability of the thin aluminum traces used. Better performance is expected in the next iteration of thermal gyro when better heater material is chosen.

The switching frequency of the heaters is set at its cut off frequency of approximately $33 \mathrm{~Hz}$. A third-order $3 \mathrm{~Hz}$ low pass filter is used to remove the $33 \mathrm{~Hz}$ modulation signal. While it is conceivable that a well designed heater can achieve a switching frequency of well above $100 \mathrm{~Hz}$ [8], the required use of a heavier gas such as the sulfur hexafluoride to improve sensitivity will limit this upward increase of heater switching frequency. The practical limit will likely be in the range of $50-100 \mathrm{~Hz}$. For this reason, the thermal gyro frequency response is not expected to go much beyond $10 \mathrm{~Hz}$. The use of higher order filters can possibly increase the thermal gyro's signal bandwidth.

The new thermal gyro described in this work switches on two heaters alternately to create bi-directional gas motions for angular rate detection. Synchronous detection is used to extract angular rate signals. In this arrangement, unwanted linear acceleration signals, along with other low frequency noise, are up-shifted in frequency and filtered. In testing the thermal gyro prototype, however, we observe very significant linear acceleration effects up to $100 \% \mathrm{~s} / \mathrm{g}$. Because Coriolis acceleration is orders of magnitude smaller than the interfering linear acceleration, very large rejection is required. We suspect the $\mathrm{XeF}_{2}$ etched cavity may be slightly asymmetrical. The residue stresses of the oxide film with metal film resistors on them may also deform the bridges and introduce additional device asymmetry. Fabricating devices with better symmetry will be an important area for future thermal gyro development.

\section{CONCLUSIONS}

A MEMS thermal gyro with unconventional device structure and sensing principle is presented in this paper. This operation of this new device has been successfully demonstrated. Only preliminary performance data have been collected on this device and more research is required to verify the competitiveness of this new technology. In spite of that, some observations can be made. Since the thermal gyroscope's device structure is identical to that of the thermal accelerometer, we can conclude that it has the potential of achieving the same robustness and low manufacture cost in the production environment. The thermal gyro's signal bandwidth will likely be limited to about $10 \mathrm{~Hz}$, and its resolution performance will be modest. The very low device cost, however, may allow this new thermal gyro technology to become one possible contender in the cost sensitive consumer market.

\section{ACKNOWLEDGEMENTS}

This work is supported by a Discovery Grant from the Natural Sciences and Engineering Research Council of Canada.

\section{REFERENCES}

[1] N. Yazdi, F. Ayazi, and K. Najafi, "Micromachined inertial sensors," Proceedings of the IEEE, vol. 86, no. 8, pp. 16401659, August 1998.

[2] W. Zhang et al. "The development of micro-gyroscope technology”, J. Micromech. Microeng. 19 (2009) 113001.

[3] P. Kollsman, "Turn indicator," US Patent 1841607, 1932.

[4] M. T. Lopiccolo, , M. A. Schaffer, and G. A. Jachyra, "Unitary fluidic angular rate sensor," US Patent 4020700, 1977.

[5] V. T. Dau, D. V. Dao, T. Shiozawa, H. Kumagai, and S. Sugiyama, "Development of a dual-axis thermal convective gas gyroscope," Journal of Micromechanics and Microengineering, vol. 16, no. 7, pp. 1301-1306, July 2006. 
[6] P. B. Chu, J. T. Chen, R. Yeh, G. Lin, J. C. P. Huang, B. A. Warneke and K. S. J. Pister, "Controlled Pulse-etching with Xenon Difluoride," Proceedings of the Ninth International Conference on Solid State Sensors and Actuators, June. 1997.

[7] A.M. Leung, J.Jones, E. Czyzewska, J.Chen and B. Woods, "Micromachined Accelerometer based on convection heat transfer", Digest, IEEE/ASME MciroElectroMechanical Systems Workshop, Heidelberg, Germany, January 25-29, 1998, pp627-630.

[8] J. Couteaud et al., "Studies and optimization of the frequency response of a micromachined thermal accelerometer", Sensors and Actuators A 147 (2008) 75-82.

\section{CONTACT}

*A.M. Leung, tel: +1-778-782-4296; aleung@,sfu.ca 